\title{
Adaptation du cahier des charges agricole aux objectifs biologiques et adhé- sion technique des agriculteurs : deux atouts pour réussir une Opération Locale Agri-Environnementale
}

\author{
A. Havet ${ }^{\mathrm{a}}$, E. Kernéïs ${ }^{\mathrm{b}}$ \\ ${ }^{a}$ Inra SADIF, BP 01, 78850 Thiverval-Grignon ; ${ }^{b}$ Inra SAD, 17450 Saint-Laurent de la Prée, \\ France
}

La mise en cuvre des Opérations Locales Agri-Environnementales se traduit par la définition de cahiers des charges dont l'objectif est la protection de l'environnement par les acteurs agricoles qui gèrent cet espace à des fins économiques. Nous nous intéressons ici à la préservation d'oiseaux limicoles sur 4700 ha de prairies naturelles humides en Vendée. Deux questions se posent : les connaissances sur l'écologie de ces oiseaux permettent-elles d'élaborer un cahier des charges efficace ? Quelles sont les conditions techniques d'adhésion des exploitants à ces propositions ? Les oiseaux préfèrent les parcelles en pâturage libre ( $43 \%$ de fréquentation), puis tournant ( $35 \%$ ) et enfin la fauche ( $16 \%$ ), alors que ces pratiques agricoles concernent respectivement 26,31 et $29 \%$ des parcelles. Nous n'avons pas observé de relation précise entre chargement, fertilisation et fréquentation des oiseaux, ce qui interroge sur l'effet des pratiques préconisées. En revanche, il semblerait que l'hydromorphie des sols puisse expliquer des fréquentations préférentielles de parcelles. Le taux de souscription au contrat est élevé ( $95 \%$ des exploitants), car les modifications de pratiques (fertilisation, modes d'exploitation) touchent seulement $18 \%$ de la surface. Seulement $26 \%$ des exploitants ont eu à accepter de fortes contraintes de localisation de pratiques pendant la négociation avec les environnementalistes. Pour accroître l'efficacité biologique, il semble falloir moduler le niveau des primes en fonction de l'intensité des adaptations de pratiques et d'une zonation écologique préalable.

\section{Étude des équilibres agri-environnementaux en zone pastorale : exemple de la Crau}

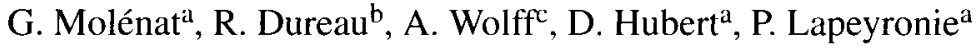

anra-UZM, 2, place Viala, 34060 Montpellier cedex $1 ;{ }^{b}$ CERPAM, 22, avenue H. Pontier, 13626 Aix-en-Provence cedex 1 ; 'CEEP, boulevard de Provence, 13310 Saint-Martin-deCrau, France

En Crau, des parcours steppiques hébergent une avifaune protégée (Ganga cata, Outarde canepetière,...$)$ menacée par les activités industrielles et agricoles. Le classement en Zone de Protection Spéciale veut sauvegarder l'avifaune, en partic grâce au pâturage ovin réputé favorable. Un programme d'étude des relations entre l'élevage et l'environnement analyse les conduites des troupeaux, leurs perspectives d'évolution ainsi que les relations entre les ovins, l'avifaune et la végétation. Tardive et de courte durée (mi avril - fin mai), la production printanière atteint $1,5 \mathrm{t} \mathrm{MS} \cdot \mathrm{ha}^{-1}$, soit $80 \%$ du total annuel. Du potentiel moyen de 800 journées de pâturage / ha / an les éleveurs mobilisent 60 à $90 \%$. Diversité de conduite des troupeaux et pressions de pâturage variables génèrent des hétérogénéités de végétation. L'avifaune en tire parti : le ganga niche plutôt dans les secteurs ras et nitrophiles, alors que la parade des outardes a lieu dans les secteurs sous pâturés. Des céréales pâturées, appréciées des outardes en hiver, compensent parfois l'insuffisance du parcours en mars (faibles production et valeur nutritive : $70 \mathrm{~g} \mathrm{MAT} \cdot \mathrm{kg}^{-1} \mathrm{MS}$ ). Une graminée pérenne, le brachypode rameux, sert de "volant de sécurité ». Des fourrages innovants comme le trèfle souterrain aux premiers résultats prometteurs ( $4 \mathrm{t} \mathrm{MS} \cdot \mathrm{ha}^{-1}$ produites au printemps ; $220 \mathrm{~g} \mathrm{MAT} \cdot \mathrm{kg}^{-1} \mathrm{MS}$ ) pourraient aussi se développer. 